\title{
Treatment of urinary schistosomiasis: methodological issues and research needs identified through a Cochrane systematic review
}

\author{
A. DANSO-APPIAH ${ }^{1,2}, *$ P. GARNER ${ }^{1}$, P. L. OLLIARO ${ }^{3}$ and J. UTZINGER ${ }^{4}$ \\ ${ }^{1}$ International Health Group, Liverpool School of Tropical Medicine, Pembroke Place, Liverpool L3 5QA, UK \\ ${ }^{2}$ Department of Public Health, Erasmus MC, University Medical Center Rotterdam, P.O. Box 2040, 3000 CA Rotterdam, \\ The Netherlands \\ ${ }^{3}$ UNICEF/UNDP/World Bank/WHO Special Programme on Research and Training in Tropical Diseases, World Health \\ Organization, 20 Avenue Appia, CH-1211 Geneva 27, Switzerland \\ ${ }^{4}$ Department of Public Health and Epidemiology, Swiss Tropical Institute, P.O. Box, CH-4002 Basel, Switzerland
}

(Received 29 October 2008; revised 23 Fanuary 2009; accepted 27 Fanuary 2009; first published online 3 Fune 2009)

SUMMARY

Guidelines recommend praziquantel (PZQ) for the treatment and control of schistosomiasis, with no real alternative. Metrifonate was still widely used against Schistosoma haematobium in the 1990s, and then withdrawn. Experimental studies and clinical trials suggest that artemisinin compounds are active against $S$. haematobium. In a Cochrane systematic review assessing the efficacy and safety of drugs for treating urinary schistosomiasis, 24 randomized controlled trials $(n=6315$ individuals) met our inclusion criteria. These trials compared a variety of single agent and combination regimens with PZQ, metrifonate or artemisinin derivatives. The review confirmed that both the standard recommended doses of PZQ (single $40 \mathrm{mg} / \mathrm{kg}$ oral dose) and metrifonate $(3 \times 7 \cdot 5-10 \mathrm{mg} / \mathrm{kg}$ oral doses administered fortnightly) are efficacious and safe in treating urinary schistosomiasis, but there is no study comparing these two regimens head-to-head. There is currently not enough evidence to evaluate artemisinin compounds. Most of the studies included in the Cochrane systematic review were insufficiently powered, lacked standardization in assessing and reporting outcomes, and had a number of methodological limitations. In this paper we discuss the implications of these findings with respect to public health and research methodology and propose priority research needs.

Key words: schistosomiasis, urinary schistosomiasis, Schistosoma haematobium, systematic review, meta-analysis, praziquantel, metrifonate, artemisinin.

\section{INTRODUCTION}

Schistosomiasis is a common parasitic disease in the tropics and subtropics. An estimated 779 million individuals are at risk of acquiring schistosomiasis and more than 200 millions were infected in mid2003 (Steinmann et al. 2006). The World Health Organization (WHO) estimates that the global burden due to schistosomiasis may be as high as $4 \cdot 5$ million disability-adjusted life years (DALYs) (WHO, 2002). However, a meta-analysis suggests that disability weights might be 2-15 times higher than those used in the global burden of disease study (King, Dickman and Tisch, 2005), and that the DALY underestimates the importance of chronic diseases like schistosomiasis (King and Bertino, 2008). This is further substantiated by the results of approaches using a quality of life questionnaire and decision-tree modelling (Jia et al. 2007; Finkelstein

* Corresponding author: Anthony Danso-Appiah, International Health Group, Liverpool School of Tropical Medicine, Pembroke Place, Liverpool L3 5QA, UK. E-mail: tdappiah@yahoo.co.uk et al. 2008). From a public health perspective, the three most important schistosome species are Schistosoma haematobium (causing urinary schistosomiasis), and S. mansoni and S.japonicum (causing intestinal schistosomiasis).

Two drugs, metrifonate and praziquantel (PZQ), have been used extensively for urinary schistosomiasis (Cioli, Pica-Mattoccia and Archer, 1995; Utzinger and Keiser, 2004). However, in the late 1990 s, metrifonate was withdrawn from the WHO model list of essential medicines because it was considered clinically, economically and operationally inferior to $\mathrm{PZQ}$ as it is only active against $S$. haematobium, requires multiple administrations, and hence is less convenient in large-scale control programmes (Feldmeier and Chitsulo, 1999). Thus, $\mathrm{PZQ}$ remains the only drug for clinical management and community-based control of schistosomiasis (Cioli, 2000; Fenwick et al. 2003; Utzinger and Keiser, 2004; Caffrey, 2007; Doenhoff, Cioli and Utzinger, 2008). Large-scale morbidity control programmes became feasible as the price of PZQ fell from approximately US\$1.0 in the 1980 s to less than US\$ 0.1 per $600 \mathrm{mg}$ tablet (Fenwick et al. 2003; 
Fenwick, Keiser and Utzinger, 2006; Doenhoff et al. 2008). Paradoxically, this also stalled investments in the discovery and development of alternative control measures, such as other drugs, vaccines and diagnostics (Utzinger et al. 2007; Bergquist, Utzinger and McManus, 2008). Research carried out over the past 15 years revealed the antischistosomal properties of artemisinin derivatives, which act especially on the young developing stages of the parasites (for a recent review see Utzinger et al. 2007) as opposed to PZQ, which acts against the adult worms and the very young schistosomula just after skin penetration (Sabah et al. 1986; Utzinger et al. 2007).

The use of PZQ has increased considerably after the 54th World Health Assembly set the target of at least $75 \%$ of school-aged children and other highrisk groups to be treated regularly with PZQ by 2010 in areas where the disease is highly endemic (WHO, 2002). At least 17 million doses of PZQ have been administered, primarily to school-aged children in selected African countries, since the launch of the 'Schistosomiasis Control Initiative' in 2003 (Fenwick et al. 2006). Relying on praziquantel alone for controlling a disease that affects millions of people is risky should resistance develop against this drug (Danso-Appiah and de Vlas, 2002; Doenhoff et al. 2008).

In the light of this, we assessed the effects of PZQ and other antischistosomal treatments by conducting a Cochrane systematic review, including comparisons between PZQ and metrifonate and trials of combination treatments. During this process, we identified a number of methodological issues relevant to the interpretation of existing data that might help researchers to design more appropriate trials in the future. The full review is available on the Cochrane Library (Danso-Appiah et al. 2008). In this paper we highlight key findings of the review, discuss implications of various methodological limitations and consider future research needs.

\section{SUMMARY OF COCHRANE SYSTEMATIC REVIEW}

\section{Inclusion criteria and search strategy}

To qualify for inclusion, a study was (1) to be controlled, randomized or quasi-randomized, enrolling individuals infected with $S$. haematobium, as determined microscopically for $S$. haematobium eggs in a standard filtrate of $10 \mathrm{ml}$ of urine or by haematuria (Feldmeier and Poggensee, 1993); and (2) to treat patients with either $\mathrm{PZQ}$, metrifonate or artemisinin derivatives. An extensive, standard search was carried out, which included MEDLINE (1966 to August 2007), EMBASE (1974 to August 2007), LILACS (1982 to August 2007), conference proceedings and contacting specialists in the field (Danso-Appiah et al. 2008).

\section{Data retrieval, quality assessment and analysis}

Eligibility and methodological quality of the identified trials were assessed by the authors and the data analysed using Review Manager 4.2 (The Cochrane Collaboration, 2003). The main outcome measure was failure rate (the proportion of individuals remaining positive for eggs in their urine at follow-up). Comparisons between groups were expressed as relative risk (RR) with $95 \%$ confidence intervals (CIs) for both individual studies and on aggregate. The secondary parameter was egg reduction rate and was analysed using weighted mean difference with standard error. The proportion of patients with adverse events was compared between treatment and control arms.

\section{Key findings of the Cochrane systematic review}

The search identified 24 randomised controlled trials that together involved 6315 participants. Table 1 summarises key characteristics of these 24 trials. When used as monotherapy, both metrifonate and PZQ showed obvious benefit in terms of parasitological outcomes (Danso-Appiah et al. 2008). One trial (120 participants) of artesunate showed no obvious benefit over placebo.

For combination treatments, one trial studied the combination of PZQ with artesunate, but there was no obvious advantage over PZQ alone.

\section{Metrifonate versus $P Z Q$ : comparisons and dose effects}

Fig. 1 summarises the failure rate of metrifonate versus $\mathrm{PZQ}$ in the five trials meeting our inclusion criteria (McMahon, 1983; Pugh and Teesdale, 1983; Wilkins and Moore, 1987; King et al. 1988; Stephenson et al. 1989).

When metrifonate was introduced, some early studies investigated a single dose of $10 \mathrm{mg} / \mathrm{kg}$ (the standard dose is $7 \cdot 5$ to $10 \mathrm{mg} / \mathrm{kg}$ three times at 14 -day intervals) versus the standard single dose of $40 \mathrm{mg} / \mathrm{kg}$ PZQ. Although the single metrifonate dose was inferior in three trials measuring failure at one to eight months, the $95 \% \mathrm{CIs}$ were too wide for statistical significance $(\mathrm{RR}=2 \cdot 31,95 \% \mathrm{CI}: 0 \cdot 91-5 \cdot 82 ; n=462$ participants). This lack of significance is due to significant heterogeneity between trials $\left(I^{2}=94 \%\right)$ likely to be associated with the duration of follow-up: the RR was 1.26 at one month of follow-up (Pugh and Teesdale, 1983), 2.23 at three months (Wilkins and Moore, 1987) and 4.62 at eight months (Stephenson et al. 1989).

There was no significant difference in failure rates when metrifonate given as multiple doses $(3 \times$ $10 \mathrm{mg} / \mathrm{kg}$ fortnightly) was compared with PZQ $(30 \mathrm{mg} / \mathrm{kg})$ in a small trial involving 54 participants (McMahon, 1983). A trial comparing three doses 
Table 1. Summary of the characteristics of randomised controlled trials included in our Cochrane systematic review (Danso-Appiah et al. 2008) evaluating antischistosomal drugs, used alone or in combination, for treating urinary schistosomiasis

\begin{tabular}{|c|c|c|c|c|c|c|c|c|c|c|c|c|c|c|}
\hline \multirow[b]{2}{*}{$\begin{array}{l}\text { Reference and } \\
\text { country where } \\
\text { trial was } \\
\text { implemented }\end{array}$} & \multirow[b]{2}{*}{$\begin{array}{l}\text { Year trial } \\
\text { was } \\
\text { conducted }\end{array}$} & \multirow[b]{2}{*}{$\mathrm{N}^{*}$} & \multirow[b]{2}{*}{$\begin{array}{l}\text { Age of } \\
\text { participants }\end{array}$} & \multirow[b]{2}{*}{$\begin{array}{l}\text { Diagnostic } \\
\text { approach** }\end{array}$} & \multirow[b]{2}{*}{$\begin{array}{l}\text { Endemicity } \\
\text { (prevalence) }\end{array}$} & \multirow[b]{2}{*}{$\begin{array}{l}\text { Sample } \\
\text { size }\end{array}$} & \multirow[b]{2}{*}{ Intervention } & \multirow[b]{2}{*}{$\begin{array}{l}\text { Outcome } \\
\text { measures }\end{array}$} & \multirow[b]{2}{*}{$\begin{array}{l}\text { Brand } \\
\text { of drug }\end{array}$} & \multirow[b]{2}{*}{$\begin{array}{l}\text { Follow-up } \\
\text { (months) }\end{array}$} & \multicolumn{4}{|c|}{ Quality assessment } \\
\hline & & & & & & & & & & & $\begin{array}{l}\text { Generation } \\
\text { of } \\
\text { allocation } \\
\text { sequence }\end{array}$ & $\begin{array}{l}\text { Allocation } \\
\text { concealment }\end{array}$ & Blinding & $\begin{array}{l}\text { Loss to } \\
\text { follow-up }\end{array}$ \\
\hline $\begin{array}{l}\text { Aden Abdi and } \\
\text { Gustafsson } \\
(1989) ; \\
\text { Somalia }\end{array}$ & $\begin{array}{l}\text { Not } \\
\text { reported }\end{array}$ & 5 & $\begin{array}{l}\text { Children: } \\
\text { mean age of } \\
14 \text { years }\end{array}$ & $\begin{array}{l}\text { Urine filtration } \\
\text { (10 ml; } \\
1 \text { specimen })\end{array}$ & Very high & 300 & $\begin{array}{l}\text { 1. Metrifonate } \\
\text { ( } 7 \cdot 5 \mathrm{mg} / \mathrm{kg} \times 3 \text { given } \\
\text { at } 2 \text {-week intervals }) \\
\text { 2. Metrifonate } \\
(5 \mathrm{mg} / \mathrm{kg} \times 3 \text { given } \\
\text { in } 1 \text { day })\end{array}$ & $\begin{array}{l}\text { 1. Cure rate } \\
\text { 2. Egg reduction } \\
\text { rate } \\
\text { 3. Adverse events }\end{array}$ & $\begin{array}{l}\text { Metrifonate } \\
\text { (Bilarcil, Bayer) }\end{array}$ & $1,2,3$ and 6 & Adequate & Adequate & $\begin{array}{l}\text { Investigators, } \\
\text { participants } \\
\text { and assessors }\end{array}$ & $33 \%$ at 2 months \\
\hline $\begin{array}{l}\text { Beasley et al. } \\
(1999) ; \\
\text { Tanzania }\end{array}$ & 1994 & 1 & $\begin{array}{l}\text { Children: } \\
7-12 \text { years }\end{array}$ & $\begin{array}{l}\text { Urine filtration } \\
\text { (10 ml; } \\
1 \text { specimen })\end{array}$ & $\operatorname{High}(56 \%)$ & 357 & $\begin{array}{l}\text { 1. } \mathrm{PZQ}(40 \mathrm{mg} / \mathrm{kg} \times 1) \\
\text { plus albendazole } \\
(400 \mathrm{mg} \times 1) \\
\text { 2. Placebo }\end{array}$ & $\begin{array}{l}\text { 1. Cure rate } \\
\text { 2. Egg reduction } \\
\text { rate } \\
\text { 3. Mean haemo- } \\
\text { globin }\end{array}$ & $\begin{array}{l}\text { PZQ (Biltricide, } \\
\text { Bayer) }\end{array}$ & 3.75 & Adequate & Unclear & $\begin{array}{l}\text { Outcome } \\
\text { assessors }\end{array}$ & $30 \%$ \\
\hline $\begin{array}{l}\text { Befidi-Mengue } \\
\text { et al. (1992); } \\
\text { Cameroon }\end{array}$ & $\begin{array}{l}\text { Not } \\
\text { reported }\end{array}$ & 1 & $\begin{array}{l}\text { Boys: } \\
6-15 \text { years }\end{array}$ & $\begin{array}{l}\text { Urine filtration } \\
\text { (10 ml; } \\
1 \text { specimen })\end{array}$ & $\begin{array}{l}\text { Not } \\
\text { reported }\end{array}$ & 436 & $\begin{array}{l}\text { 1. } \mathrm{PZQ}(40 \mathrm{mg} / \mathrm{kg} \times 1) \\
\text { 2. Placebo }\end{array}$ & $\begin{array}{l}\text { 1. Anaemia } \\
\text { 2. Physical growth } \\
\text { 3. Haematuria } \\
\text { 4. Proteinuria }\end{array}$ & Not stated & 6 & Unclear & Unclear & Unclear & Unclear \\
\hline $\begin{array}{l}\text { Borrmann } \\
\text { et al. (2001); } \\
\text { Gabon }\end{array}$ & $\begin{array}{l}\text { Not } \\
\text { reported }\end{array}$ & 3 & $\begin{array}{l}\text { Children: } \\
\text { 5-13 years }\end{array}$ & $\begin{array}{l}\text { Urine filtration } \\
\text { (10 ml; } \\
2 \text { specimens })\end{array}$ & $\begin{array}{l}\text { Very high } \\
(80 \%)\end{array}$ & 300 & $\begin{array}{l}\text { 1. } \mathrm{PZQ}(40 \mathrm{mg} / \mathrm{kg} \times 1) \\
\text { 2. Artesunate }(4 \mathrm{mg} / \\
\mathrm{kg} / \text { day for } 3 \mathrm{days}) \\
\text { 3. } \mathrm{PZQ}(40 \mathrm{mg} / \mathrm{kg} \times 1) \\
\text { plus artesunate } \\
(4 \mathrm{mg} / \mathrm{kg} / \text { day over } 3 \\
\text { days }) \\
\text { 4. } \text { Placebo }\end{array}$ & $\begin{array}{l}\text { 1. Cure rate } \\
\text { 2. Adverse events } \\
\text { 3. Resolution of } \\
\text { haematuria } \\
\text { 4. Egg reduction } \\
\text { rate }\end{array}$ & Not stated & 8 & Adequate & Adequate & $\begin{array}{c}\text { Participants and } \\
\text { investigators }\end{array}$ & $1 \cdot 3 \%$ \\
\hline $\begin{array}{l}\text { Davis et al. } \\
\text { (1981); } \\
\text { Zambia }\end{array}$ & $\begin{array}{l}\text { Not } \\
\text { reported }\end{array}$ & 1 & $\begin{array}{l}\text { Children } \\
\text { and young } \\
\text { adults: } \\
7-22 \text { years }\end{array}$ & $\begin{array}{l}\text { Urine filtration } \\
\text { (10 ml; } \\
3 \text { specimens })\end{array}$ & $\begin{array}{l}\text { Not } \\
\text { reported }\end{array}$ & 79 & $\begin{array}{l}\text { 1. } \mathrm{PZQ} \\
(20 \mathrm{mg} / \mathrm{kg} \times 1) \\
\text { 2. } \mathrm{PZQ} \\
(30 \mathrm{mg} / \mathrm{kg} \times 1) \\
\text { 3. } \mathrm{PZQ}(40 \mathrm{mg} / \mathrm{kg} \times 1) \\
\text { 4. } \mathrm{PZQ} \\
(20 \mathrm{mg} / \mathrm{kg} \times 2) \\
\text { 5. } \\
\text { Placebo }\end{array}$ & $\begin{array}{l}\text { 1. Adverse events } \\
\text { 2. Cure rate } \\
\text { 3. Mean } \\
\text { haemoglobin }\end{array}$ & Not stated & $\begin{array}{l}1,6,9 \text { and } \\
12\end{array}$ & Adequate & Adequate & $\begin{array}{l}\text { Participants, } \\
\text { investigators } \\
\text { and outcome } \\
\text { assessors }\end{array}$ & $\begin{array}{l}7 \cdot 6 \% \text { and } 16 \cdot 5 \% \\
\text { at } 6 \text { and } 12 \\
\text { months, } \\
\text { respectively }\end{array}$ \\
\hline $\begin{array}{l}\text { Doehring et al. } \\
\text { (1985); } \\
\text { Sudan }\end{array}$ & $\begin{array}{l}\text { Not } \\
\text { reported }\end{array}$ & 1 & $\begin{array}{l}\text { Boys: } \\
6-13 \text { years }\end{array}$ & $\begin{array}{l}\text { Urine filtration } \\
\text { (10 ml; } \\
3 \text { specimens })\end{array}$ & Very high & 182 & $\begin{array}{l}\text { 1. PZQ }(40 \mathrm{mg} / \mathrm{kg} \times 1) \\
\text { 2. Metrifonate }(10 \mathrm{mg} / \\
\mathrm{kg} \times 3 \text { given at } \\
2 \text {-week intervals }) \\
\text { 3. Placebo }\end{array}$ & $\begin{array}{l}\text { 1. Egg reduction } \\
\text { rate } \\
\text { 2. Proteinuria }\end{array}$ & Not stated & 1 & Unclear & Unclear & Unclear & $0 \%$ \\
\hline $\begin{array}{l}\text { Jewsbury and } \\
\text { Cooke } \\
\text { (1976); } \\
\text { Zimbabwe }\end{array}$ & $\begin{array}{l}\text { Not } \\
\text { reported }\end{array}$ & 4 & $\begin{array}{l}\text { Children } \\
\text { (age not } \\
\text { specified) }\end{array}$ & $\begin{array}{l}\text { Urine filtration } \\
\text { (10 ml; } \\
1 \text { specimen vs. } \\
3 \text { specimens) }\end{array}$ & $\begin{array}{l}\text { Very high } \\
(80 \%)\end{array}$ & 179 & $\begin{array}{l}\text { 1. Metrifonate } \\
(7.5 \mathrm{mg} / \mathrm{kg} \times 3 \text { given } \\
\text { at } 2 \text {-week intervals }) \\
\text { 2. Placebo } \\
\text { (Third arm excluded } \\
\text { from current review) }\end{array}$ & $\begin{array}{l}\text { 1. Cure rate } \\
\text { 2. Egg reduction } \\
\text { rate } \\
\text { 3. Adverse events }\end{array}$ & $\begin{array}{l}\text { Metrifonate } \\
\text { (Bilarcil, Bayer) }\end{array}$ & $1 \cdot 2,7$ and 16 & Adequate & Unclear & Unclear & Not reported \\
\hline $\begin{array}{l}\text { Jinabhai et al. } \\
\text { (2001); South } \\
\text { Africa }\end{array}$ & $\begin{array}{l}\text { Not } \\
\text { reported }\end{array}$ & 11 & $\begin{array}{l}\text { Children: } \\
\text { 8-10 years }\end{array}$ & Not stated & High & 268 & $\begin{array}{l}\text { 1. PZQ }(40 \mathrm{mg} / \mathrm{kg} \times 1) \\
\text { plus albendazole } \\
(400 \mathrm{mg} \times 1) \\
\text { 2. Placebo } \\
\text { (Another arm consisted } \\
\text { of albendazole } \\
(400 \mathrm{mg} \times 1) \\
\text { for intestinal } \\
\text { helminths) }\end{array}$ & 1. Cure rate & Not stated & 4 & Unclear & Unclear & Unclear & Not reported \\
\hline
\end{tabular}




\begin{tabular}{|c|c|c|c|c|c|c|c|c|c|c|c|c|c|c|}
\hline \multirow[b]{2}{*}{$\begin{array}{l}\text { Reference and } \\
\text { country where } \\
\text { trial was } \\
\text { implemented }\end{array}$} & \multirow[b]{2}{*}{$\begin{array}{l}\text { Year trial } \\
\text { was } \\
\text { conducted }\end{array}$} & \multirow[b]{2}{*}{$\mathrm{N}^{*}$} & \multirow[b]{2}{*}{$\begin{array}{l}\text { Age of } \\
\text { participants }\end{array}$} & \multirow[b]{2}{*}{$\begin{array}{l}\text { Diagnostic } \\
\text { approach** }\end{array}$} & \multirow[b]{2}{*}{$\begin{array}{l}\text { Endemicity } \\
\text { (prevalence) }\end{array}$} & \multirow[b]{2}{*}{$\begin{array}{l}\text { Sample } \\
\text { size }\end{array}$} & \multirow[b]{2}{*}{ Intervention } & \multirow[b]{2}{*}{$\begin{array}{l}\text { Outcome } \\
\text { measures }\end{array}$} & \multirow[b]{2}{*}{$\begin{array}{l}\text { Brand } \\
\text { of drug }\end{array}$} & \multirow[b]{2}{*}{$\begin{array}{l}\text { Follow-up } \\
\text { (months) }\end{array}$} & \multicolumn{4}{|c|}{ Quality assessment } \\
\hline & & & & & & & & & & & $\begin{array}{l}\text { Generation } \\
\text { of } \\
\text { allocation } \\
\text { sequence }\end{array}$ & $\begin{array}{l}\text { Allocation } \\
\text { concealment }\end{array}$ & Blinding & $\begin{array}{l}\text { Loss to } \\
\text { follow-up }\end{array}$ \\
\hline $\begin{array}{l}\text { Kardaman } \\
\text { et al. }(1985) \text {; } \\
\text { Sudan }\end{array}$ & $\begin{array}{l}\text { Not } \\
\text { reported }\end{array}$ & $\begin{array}{l}\text { Not } \\
\text { reported }\end{array}$ & $\begin{array}{l}\text { Children: } \\
7-11 \text { years }\end{array}$ & $\begin{array}{l}\text { Urine filtration } \\
\text { (10 ml; } \\
2 \text { specimens })\end{array}$ & $\begin{array}{l}\text { Not } \\
\text { reported }\end{array}$ & 237 & $\begin{array}{l}\text { 1. } \mathrm{PZQ}(40 \mathrm{mg} / \mathrm{kg} \times 1) \\
\text { 2. } \mathrm{PZQ}(20 \mathrm{mg} / \mathrm{kg} \times 2)\end{array}$ & $\begin{array}{l}\text { 1. Adverse events } \\
\text { 2. Cure rate } \\
\text { 3. Egg reduction } \\
\text { rate }\end{array}$ & Not reported & $\begin{array}{l}1,3,6 \text { and } \\
12\end{array}$ & Unclear & Unclear & Unclear & $7 \cdot 3 \%$ \\
\hline $\begin{array}{l}\text { King et al. } \\
\text { (1988); } \\
\text { Kenya }\end{array}$ & $\begin{array}{l}\text { Not } \\
\text { reported }\end{array}$ & $\begin{array}{l}\text { Not } \\
\text { reported }\end{array}$ & $\begin{array}{l}\text { Children } \\
\text { and young } \\
\text { adults: } \\
4-21 \text { years }\end{array}$ & $\begin{array}{l}\text { Urine filtration } \\
\text { (10 ml; } \\
2 \text { specimens })\end{array}$ & $\begin{array}{l}\text { Not } \\
\text { reported }\end{array}$ & 2628 & $\begin{array}{l}\text { 1. Metrifonate }(10 \mathrm{mg} / \\
\mathrm{kg} \times 3 \text { given at } \\
\text { 4-month intervals }) \\
\text { 2. PZQ }(40 \mathrm{mg} / \mathrm{kg} \times 1)\end{array}$ & $\begin{array}{l}\text { 1. Haematuria } \\
\text { 2. Proteinuria } \\
\text { (Other arms } \\
\text { not relevant } \\
\text { for current } \\
\text { review, hence } \\
\text { were excluded) }\end{array}$ & $\begin{array}{l}\text { Metrifonate } \\
\text { (Bilarcil, Bayer); } \\
\text { PZQ (Biltricide } \\
\text { Bayer) }\end{array}$ & 12 & Adequate & Unclear & $\begin{array}{l}\text { Participants and } \\
\text { assessors }\end{array}$ & $23 \%$ \\
\hline $\begin{array}{l}\text { King et al. } \\
\text { (1989); } \\
\text { Kenya }\end{array}$ & $\begin{array}{l}\text { Not } \\
\text { reported }\end{array}$ & $\begin{array}{l}\text { Not } \\
\text { reported }\end{array}$ & $\begin{array}{l}\text { Children } \\
\text { and adults } \\
\text { (age not } \\
\text { specified) }\end{array}$ & $\begin{array}{l}\text { Urine filtration } \\
\text { (10 ml; } \\
2 \text { specimens) }\end{array}$ & Very high & 280 & $\begin{array}{l}\text { 1. } \mathrm{PZQ}(40 \mathrm{mg} / \mathrm{kg} \times 1) \\
\text { 2. } \mathrm{PZQ}(30 \mathrm{mg} / \mathrm{kg} \times 1) \\
\text { 3. } \mathrm{PZQ}(20 \mathrm{mg} / \mathrm{kg} \times 1) \\
\text { 4. } \mathrm{PZQ}(10 \mathrm{mg} / \mathrm{kg} \times 1)\end{array}$ & $\begin{array}{l}\text { 1. Cure rate } \\
\text { 2. Egg reduction } \\
\text { rate } \\
\text { 3. Haematuria } \\
\text { 4. Proteinuria }\end{array}$ & Not reported & 3 & Adequate & Unclear & Unclear & $14 \%$ \\
\hline $\begin{array}{l}\text { King et al. } \\
(2002) ; \\
\text { Kenya }\end{array}$ & 1993 & 2 & $\begin{array}{l}\text { Children } \\
\text { and young } \\
\text { adults: } \\
4-23 \text { years }\end{array}$ & $\begin{array}{l}\text { Urine filtration } \\
\text { (10 ml; } \\
2 \text { specimens })\end{array}$ & $\begin{array}{l}\text { Very high } \\
(80 \%)\end{array}$ & 291 & $\begin{array}{l}\text { 1. } \mathrm{PZQ}(40 \mathrm{mg} / \mathrm{kg} \times 1) \\
\text { 2. } \mathrm{PZQ}(20 \mathrm{mg} / \mathrm{kg} \times 1)\end{array}$ & $\begin{array}{l}\text { 1. Cure rate } \\
\text { 2. Egg reduction } \\
\text { rate } \\
\text { 3. Haematuria } \\
\text { 4. Proteinuria }\end{array}$ & Not reported & $2 \cdot 5$ and 9 & Adequate & Unclear & $\begin{array}{l}\text { Clinicians and } \\
\text { assessors }\end{array}$ & $31 \%$ \\
\hline $\begin{array}{l}\text { McMahon and } \\
\text { Kolstrup } \\
(1979) ; \\
\text { Tanzania }\end{array}$ & $\begin{array}{l}\text { Not } \\
\text { reported }\end{array}$ & 1 & $\begin{array}{l}\text { Children: } \\
7-15 \text { years }\end{array}$ & $\begin{array}{l}\text { Urine filtration } \\
\text { (10 ml; } \\
3 \text { specimens })\end{array}$ & $\begin{array}{l}\text { Not } \\
\text { reported }\end{array}$ & 183 & $\begin{array}{l}\text { 1. } \mathrm{PZQ}(30 \mathrm{mg} / \mathrm{kg} \times 1) \\
\text { 2. } \mathrm{PZQ}(40 \mathrm{mg} / \mathrm{kg} \times 1) \\
\text { 3. PZQ }(20 \mathrm{mg} / \mathrm{kg} \times 2) \\
\text { 4. Placebo }\end{array}$ & $\begin{array}{l}\text { 1. Cure rate } \\
\text { 2. Egg reduction } \\
\text { rate } \\
\text { 3. Adverse events }\end{array}$ & $\begin{array}{l}\text { PZQ (Biltricide, } \\
\text { Bayer) }\end{array}$ & 1,3 and 6 & Unclear & Unclear & Unclear & $\begin{array}{l}31 \%, 32 \% \text { and } \\
36 \% \text { at } 1,3 \text { and } \\
6 \text { months, } \\
\text { respectively }\end{array}$ \\
\hline $\begin{array}{l}\text { McMahon } \\
(1983) ; \\
\text { Tanzania }\end{array}$ & $\begin{array}{l}\text { Not } \\
\text { reported }\end{array}$ & 1 & $\begin{array}{l}\text { Children } \\
\text { and adults } \\
\text { (age not } \\
\text { specified) }\end{array}$ & $\begin{array}{l}\text { Urine filtration } \\
\text { (10 ml; } \\
3 \text { specimens })\end{array}$ & $\begin{array}{l}\text { Not } \\
\text { reported }\end{array}$ & 90 & $\begin{array}{l}\text { 1. Metrifonate }(10 \mathrm{mg} / \\
\mathrm{kg} \times 3 \text { given at } \\
2 \text {-week intervals }) \\
\text { 2. PZQ }(30 \mathrm{mg} / \mathrm{kg} \times 1) \\
\text { (Third arm consisting } \\
\text { of niradazole was } \\
\text { excluded) }\end{array}$ & $\begin{array}{l}\text { 1. Cure rate } \\
\text { 2. Egg reduction } \\
\text { rate } \\
\text { 3. Adverse events }\end{array}$ & Not reported & 2 and 4 & Unclear & Unclear & Unclear & $\begin{array}{l}14 \% \text { and } 31 \% \text { at } \\
2 \text { and } 4 \text { months, } \\
\text { respectively }\end{array}$ \\
\hline $\begin{array}{l}\text { Olds et al. } \\
\text { (1999); } \\
\text { Kenya }\end{array}$ & $\begin{array}{l}\text { Not } \\
\text { reported }\end{array}$ & 1 & $\begin{array}{l}\text { Children: } \\
\text { 4-18 years }\end{array}$ & $\begin{array}{l}\text { Urine filtration } \\
(10 \mathrm{ml} ; \\
2 \text { specimen } v s . \\
10 \mathrm{ml} ; \\
1 \text { specimen })\end{array}$ & Very high & 380 & $\begin{array}{l}\text { 1. } \mathrm{PZQ}(40 \mathrm{mg} / \mathrm{kg} \times 1) \\
\text { plus } \\
\text { albendazole } \\
(400 \mathrm{mg} \times 1) \\
\text { 2. } \mathrm{PZQ} \text { plus } \\
\text { albendazole } \\
\text { placebo } \\
\text { 3. Albendazole plus } \\
\text { PZQ placebo } \\
\text { 4. Both placebos }\end{array}$ & $\begin{array}{l}\text { 1. Physical growth } \\
\text { 2. Haemoglobin } \\
\text { 3. Failure rate } \\
\text { 4. Egg reduction } \\
\text { rate } \\
\text { 5. Adverse effects }\end{array}$ & Not reported & $\begin{array}{l}1 \cdot 5,3,6 \text { and } \\
12\end{array}$ & Adequate & Adequate & $\begin{array}{l}\text { Participants and } \\
\text { assessors }\end{array}$ & $\begin{array}{l}1 \%, 1 \%, 10 \% \\
\text { and } 17 \% \text { at } 1 \cdot 5, \\
3,6 \text { and } 12 \\
\text { months, } \\
\text { respectively }\end{array}$ \\
\hline $\begin{array}{l}\text { Omer (1981); } \\
\text { Sudan }\end{array}$ & $1978-79$ & 1 & $\begin{array}{l}\text { Children } \\
\text { (>8 years) } \\
\text { and adults }\end{array}$ & $\begin{array}{l}\text { Urine filtration } \\
\text { (10 ml; } \\
1 \text { specimen })\end{array}$ & Very high & 153 & $\begin{array}{l}\text { 1. } \mathrm{PZQ}(30 \mathrm{mg} / \mathrm{kg} \times 1) \\
\text { 2. } \mathrm{PZQ}(40 \mathrm{mg} / \mathrm{kg} \times 1) \\
\text { 3. } \mathrm{PZQ}(20 \mathrm{mg} / \mathrm{kg} \times 2)\end{array}$ & $\begin{array}{l}\text { 1. Adverse events } \\
\text { 2. Cure rate } \\
\text { 3. Egg reduction } \\
\text { rate }\end{array}$ & $\begin{array}{l}\text { PZQ (Biltricide, } \\
\text { Bayer) }\end{array}$ & 6 & Unclear & Unclear & Unclear & $20 \%$ \\
\hline $\begin{array}{l}\text { Oyediran et al. } \\
\text { (1981); } \\
\text { Nigeria }\end{array}$ & $\begin{array}{l}\text { Not } \\
\text { reported }\end{array}$ & 1 & $\begin{array}{l}\text { Children: } \\
\text { 9-16 years }\end{array}$ & $\begin{array}{l}\text { Urine filtration } \\
\text { (10 ml; } \\
3 \text { specimens })\end{array}$ & $\begin{array}{l}\text { Light to } \\
\text { moderate }\end{array}$ & 90 & $\begin{array}{l}\text { 1. } \mathrm{PZQ}(30 \mathrm{mg} / \mathrm{kg} \times 1) \\
\text { 2. } \mathrm{PZQ}(40 \mathrm{mg} / \mathrm{kg} \times 1) \\
\text { 3. } \mathrm{PZQ}(20 \mathrm{mg} / \mathrm{kg} \times 2) \\
\text { 4. Placebo }\end{array}$ & $\begin{array}{l}\text { 1. Egg reduction } \\
\text { rate } \\
\text { 2. Adverse events }\end{array}$ & $\begin{array}{l}\text { PZQ (Biltricide, } \\
\text { Bayer) }\end{array}$ & 1,3 and 6 & Unclear & Unclear & Unclear & $\begin{array}{l}31 \cdot 7 \%, 32 \cdot 3 \% \\
\text { and } 36 \% \text { at } 1 \text {, } \\
\text { 3and } 6 \text { months, } \\
\text { respectively }\end{array}$ \\
\hline
\end{tabular}




\begin{tabular}{|c|c|c|c|c|c|c|c|c|c|c|c|c|c|c|}
\hline \multirow[b]{2}{*}{$\begin{array}{l}\text { Reference and } \\
\text { country where } \\
\text { trial was } \\
\text { implemented }\end{array}$} & \multirow[b]{2}{*}{$\begin{array}{l}\text { Year trial } \\
\text { was } \\
\text { conducted }\end{array}$} & \multirow[b]{2}{*}{$\mathrm{N}^{*}$} & \multirow[b]{2}{*}{$\begin{array}{l}\text { Age of } \\
\text { participants }\end{array}$} & \multirow[b]{2}{*}{$\begin{array}{l}\text { Diagnostic } \\
\text { approach** }\end{array}$} & \multirow[b]{2}{*}{$\begin{array}{l}\text { Endemicity } \\
\text { (prevalence) }\end{array}$} & \multirow[b]{2}{*}{$\begin{array}{l}\text { Sample } \\
\text { size }\end{array}$} & \multirow[b]{2}{*}{ Intervention } & \multirow[b]{2}{*}{$\begin{array}{l}\text { Outcome } \\
\text { measures }\end{array}$} & \multirow[b]{2}{*}{$\begin{array}{l}\text { Brand } \\
\text { of drug }\end{array}$} & \multirow[b]{2}{*}{$\begin{array}{l}\text { Follow-up } \\
\text { (months) }\end{array}$} & \multicolumn{4}{|c|}{ Quality assessment } \\
\hline & & & & & & & & & & & $\begin{array}{l}\text { Generation } \\
\text { of } \\
\text { allocation } \\
\text { sequence }\end{array}$ & $\begin{array}{l}\text { Allocation } \\
\text { concealment }\end{array}$ & Blinding & $\begin{array}{l}\text { Loss to } \\
\text { follow-up }\end{array}$ \\
\hline $\begin{array}{l}\text { Pugh and } \\
\text { Teesdale } \\
\text { (1983); } \\
\text { Malawi }\end{array}$ & $\begin{array}{l}\text { Not } \\
\text { reported }\end{array}$ & $\begin{array}{l}\text { Not } \\
\text { reported }\end{array}$ & $\begin{array}{l}\text { Children: } \\
5-18 \text { years }\end{array}$ & $\begin{array}{l}\text { Urine filtration } \\
\text { (10 ml; } \\
1 \text { specimen })\end{array}$ & $\begin{array}{l}\text { Not } \\
\text { reported }\end{array}$ & 600 & $\begin{array}{l}\text { 1. } \mathrm{PZQ}(40 \mathrm{mg} / \mathrm{kg} \times 1) \\
\text { 2. Metrifonate }(10 \mathrm{mg} / \\
\mathrm{kg} \times 1) \\
\text { 3. Placebo }\end{array}$ & $\begin{array}{l}\text { 1. Cure rate } \\
\text { 2. Egg reduction } \\
\text { rate } \\
\text { 3. Adverse events }\end{array}$ & $\begin{array}{l}\text { Metrifonate } \\
\text { (Bilarcil, Bayer); } \\
\text { PZQ (Biltricide, } \\
\text { Bayer) }\end{array}$ & 1,3 and 6 & Inadequate & Unclear & $\begin{array}{l}\text { Participants, } \\
\text { clinicians and } \\
\text { assessors }\end{array}$ & $3 \%$ at 1 month \\
\hline $\begin{array}{l}\text { Rey et al. } \\
\text { (1983); Niger }\end{array}$ & $\begin{array}{l}\text { Not } \\
\text { reported }\end{array}$ & 3 & $\begin{array}{l}\text { Children } \\
\text { and adults } \\
\text { (age not } \\
\text { specified) }\end{array}$ & $\begin{array}{l}\text { Urine filtration } \\
\text { (10 ml; } \\
1 \text { specimen })\end{array}$ & $\operatorname{High}(50 \%)$ & 103 & $\begin{array}{l}\text { 1. Metrifonate }(10 \mathrm{mg} / \\
\mathrm{kg} \times 1) \\
\text { 2. Metrifonate }(10 \mathrm{mg} / \\
\mathrm{kg} \times 2 \text { given } \\
\text { fortnightly }) \\
\text { 3. Metrifonate }(10 \mathrm{mg} / \\
\mathrm{kg} \times 3 \text { given } \\
\text { fortnightly })\end{array}$ & $\begin{array}{l}\text { 1. Cure rate } \\
\text { 2. Egg reduction } \\
\text { rate }\end{array}$ & Not reported & 1,3 and 6 & Adequate & Unclear & Unclear & $\begin{array}{l}9 \cdot 7 \%, 6 \cdot 4 \% \text { and } \\
8 \cdot 1 \% \text { at } 1,3 \\
\text { and } 6 \text { months, } \\
\text { respectively }\end{array}$ \\
\hline $\begin{array}{l}\text { Rey, Nouhou } \\
\text { and Sellin } \\
\text { (1984); Niger }\end{array}$ & $\begin{array}{l}\text { Not } \\
\text { reported }\end{array}$ & 3 & $\begin{array}{l}\text { Children } \\
\text { and adults } \\
\text { (age not } \\
\text { specified) }\end{array}$ & $\begin{array}{l}\text { Urine filtration } \\
\text { (10 ml; } \\
1 \text { specimen })\end{array}$ & $\begin{array}{l}\text { Not } \\
\text { reported }\end{array}$ & 286 & $\begin{array}{l}\text { 1. Metrifonate }(10 \mathrm{mg} / \\
\mathrm{kg} \times 1) \\
\text { 2. Metrifonate }(10 \mathrm{mg} / \\
\mathrm{kg} \times 2 \text { given } \\
\text { fortnightly } \\
\text { 3. Metrifonate }(10 \mathrm{mg} / \\
\mathrm{kg} \times 3 \text { given } \\
\text { fortnightly })\end{array}$ & $\begin{array}{l}\text { 1. Cure rate } \\
\text { 2. Egg reduction } \\
\text { rate }\end{array}$ & Not reported & 2 and 4 & Unclear & Unclear & Unclear & $\begin{array}{l}50 \% \text { and } 38 \cdot 8 \% \\
\text { at } 2 \text { and } 4 \\
\text { months, } \\
\text { respectively }\end{array}$ \\
\hline $\begin{array}{l}\text { Stephenson } \\
\text { et al. (1985); } \\
\text { Kenya }\end{array}$ & $\begin{array}{l}\text { Not } \\
\text { reported }\end{array}$ & 1 & $\begin{array}{l}\text { Children: } \\
6-15 \text { years }\end{array}$ & $\begin{array}{l}\text { Urine filtration } \\
(10 \mathrm{ml} \text { of urine } \\
\text { adjusted for whole } \\
\text { volume })\end{array}$ & $\begin{array}{l}\text { Moderate } \\
(46 \%)\end{array}$ & 400 & $\begin{array}{l}\text { 1. Metrifonate } \\
(7 \cdot 5 \mathrm{mg} / \mathrm{kg} \times 3 \text { given } \\
\text { fortnightly) } \\
\text { 2. Placebo }\end{array}$ & $\begin{array}{l}\text { 1. Cure rate } \\
\text { 2. Splenomegaly } \\
\text { 3. Hepatomegaly } \\
\text { 4. Egg reduction } \\
\text { rate } \\
\text { 5. Haemoglobin } \\
\text { 6. Anthropometric } \\
\text { measurements }\end{array}$ & Not reported & 8 & Unclear & Unclear & Assessors & Not reported \\
\hline $\begin{array}{l}\text { Stephenson } \\
\text { et al. }(1989) \text {; } \\
\text { Kenya }\end{array}$ & $\begin{array}{l}\text { Not } \\
\text { reported }\end{array}$ & $\begin{array}{l}\text { Not } \\
\text { reported }\end{array}$ & $\begin{array}{l}\text { Children } \\
\text { (age not } \\
\text { specified) }\end{array}$ & $\begin{array}{l}\text { Urine filtration } \\
(10 \mathrm{ml} \text { of urine } \\
\text { adjusted for whole } \\
\text { volume })\end{array}$ & $\begin{array}{l}\text { Light to } \\
\text { moderate }\end{array}$ & 347 & $\begin{array}{l}\text { 1. Metrifonate }(10 \mathrm{mg} / \\
\mathrm{kg} \times 1) \\
\text { 2. } \mathrm{PZQ}(40 \mathrm{mg} / \mathrm{kg} \times 1) \\
\text { 3. Placebo }\end{array}$ & $\begin{array}{l}\text { 1. Cure rate } \\
\text { 2. Egg reduction } \\
\text { rate } \\
\text { 3. Physical growth }\end{array}$ & Not reported & 8 & Unclear & Unclear & Assessors & $10 \%$ \\
\hline $\begin{array}{l}\text { Taylor, } \\
\text { Murare and } \\
\text { Manomano } \\
\text { (1988); } \\
\text { Zimbabwe }\end{array}$ & $\begin{array}{l}\text { Not } \\
\text { reported }\end{array}$ & 1 & $\begin{array}{l}\text { Children: } \\
10-15 \text { years }\end{array}$ & $\begin{array}{l}\text { Urine filtration } \\
\text { (10 ml; } \\
3 \text { specimens) }\end{array}$ & $\begin{array}{l}\text { Very high } \\
(77 \%)\end{array}$ & 373 & $\begin{array}{l}\text { 1. } \mathrm{PZQ}(10 \mathrm{mg} / \mathrm{kg} \times 1) \\
\text { 2. } \mathrm{PZQ}(20 \mathrm{~m} / \mathrm{kg} \times 1) \\
\text { 3. } \mathrm{PZQ}(30 \mathrm{~m} / \mathrm{kg} \times 1) \\
\text { 4. } \mathrm{PZQ}(40 \mathrm{mg} / \mathrm{kg} \times 1) \\
\text { 5. Placebo }\end{array}$ & $\begin{array}{l}\text { 1. Cure rate } \\
\text { 2. Egg reduction } \\
\text { rate }\end{array}$ & Not reported & 1,3 and 6 & Unclear & Unclear & Assessors & $\begin{array}{l}\text { No losses } \\
\text { reported }\end{array}$ \\
\hline $\begin{array}{l}\text { Wilkins and } \\
\text { Moore } \\
\text { (1987); } \\
\text { Gambia }\end{array}$ & $\begin{array}{l}\text { Not } \\
\text { reported }\end{array}$ & 3 & $\begin{array}{l}\text { Children: } \\
2-19 \text { years }\end{array}$ & $\begin{array}{l}\text { Urine filtration } \\
\text { (10 ml; } \\
3 \text { specimens })\end{array}$ & Very high & 184 & $\begin{array}{l}\text { 1. } \mathrm{PZQ}(40 \mathrm{mg} / \mathrm{kg} \times 1) \\
\text { 2. } \mathrm{PZQ}(20 \mathrm{mg} / \mathrm{kg} \times 1) \\
\text { 3. } \mathrm{PZQ}(10 \mathrm{mg} / \mathrm{kg} \times 1) \\
\text { 4. Metrifonate }(10 \mathrm{mg} / \\
\mathrm{kg} \times 1) \\
\text { 5. Metrifonate }(10 \mathrm{mg} / \\
\mathrm{kg} \times 1) \text { plus } \mathrm{PZQ} \\
(10 \mathrm{mg} / \mathrm{kg} \times 1)\end{array}$ & $\begin{array}{l}\text { 1. Egg reduction } \\
\text { rate } \\
\text { 2. Adverse events }\end{array}$ & Not reported & 3 & Adequate & Unclear & Assessors & $\begin{array}{l}\text { No losses } \\
\text { reported }\end{array}$ \\
\hline
\end{tabular}

* $\mathrm{N}$ - Number of communities involved in the trial.

** For urine filtration, $10 \mathrm{ml} ; 3$ specimen $v s .10 \mathrm{ml} ; 1$ specimen means pre- and post-treatment diagnosis varied; i.e. pre-treatment diagnosis involved $10 \mathrm{ml}$ and 3 specimen but $10 \mathrm{ml}$ and 1 specimen for post-treatment assessment. 


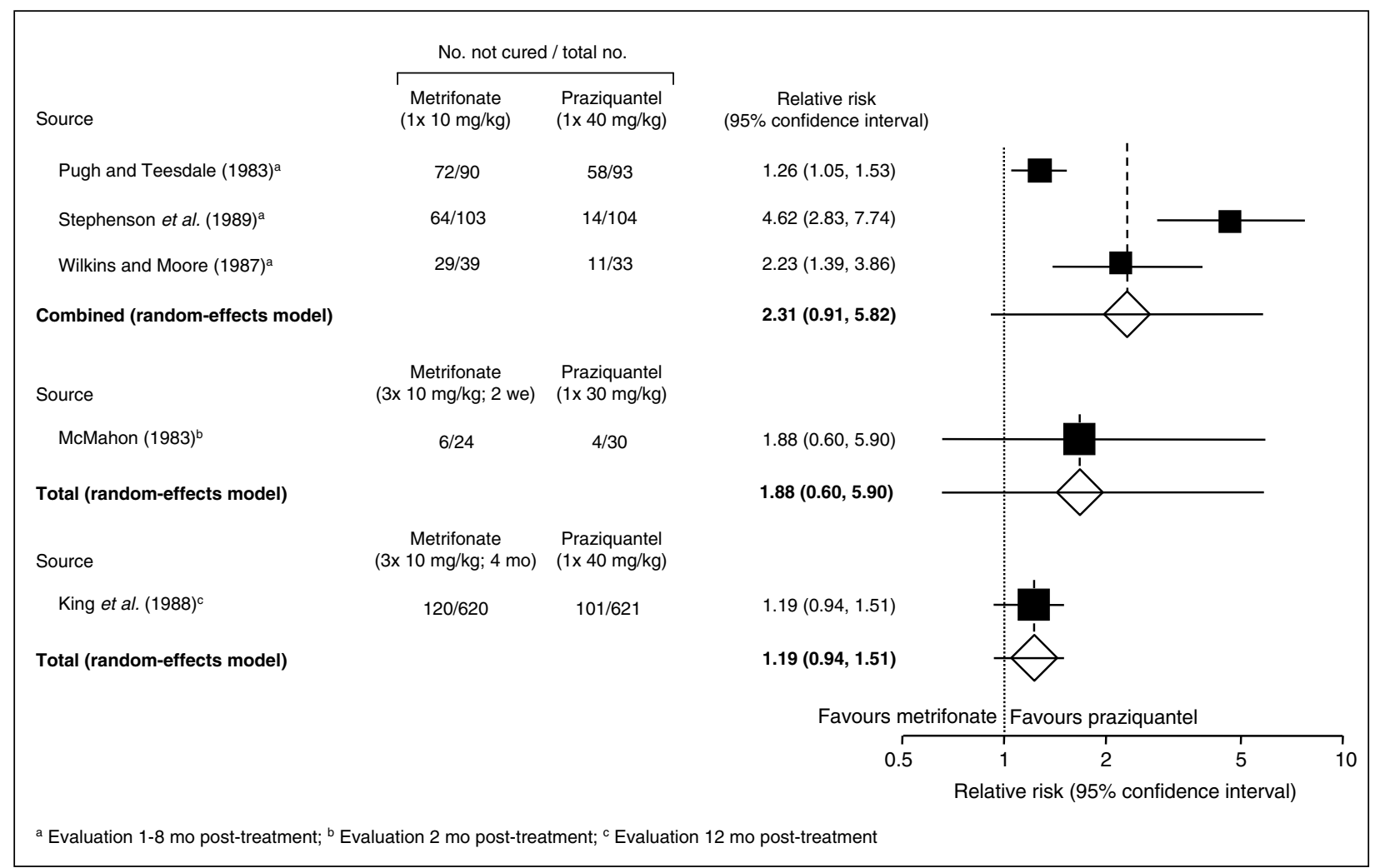

Fig. 1. Risk ratio estimates (combined or total) of randomized controlled trial(s) of metrifonate (different regimens) versus $\mathrm{PZQ}$ (single dose, $30 \mathrm{mg} / \mathrm{kg}$ or $40 \mathrm{mg} / \mathrm{kg}$ ) against $S$. haematobium. Rectangles represent risk ratios and sizes of the rectangles denote the weight given to each trial in the meta-analysis. Diamond and vertical broken line indicate combined (total) relative risk (RR). Horizontal lines indicate $95 \%$ confidence intervals. The dashed vertical line is the null value ( $\mathrm{RR}=1$; neither favouring metrifonate nor $\mathrm{PZQ})$. Abbreviations: mo, month; we, week.

of metrifonate at $10 \mathrm{mg} / \mathrm{kg}$ given once every four months with the standard $40 \mathrm{mg} / \mathrm{kg}$ PZQ (single dose) in school-aged children in Kenya detected no difference overall, but metrifonate was superior in the subgroup of children with a heavy infection $(\mathrm{RR}=0 \cdot 88$, 95\% CI : 0.80-0.96; $n=615$ participants). However, as the subgroup was stratified after randomisation, this result should be interpreted with caution. Both metrifonate (two and three doses of $10 \mathrm{mg} / \mathrm{kg}$ ) and PZQ (single dose $40 \mathrm{mg} / \mathrm{kg}$ ) led to very high reductions in egg excretion $(>98 \%)$ in two trials (McMahon, 1983; Doehring et al. 1985).

One trial ( $n=54$ participants) compared adverse events and reported similar minor events between metrifonate $(3 \times 10 \mathrm{mg} / \mathrm{kg})$ and PZQ $(30 \mathrm{mg} / \mathrm{kg})$; no serious adverse events were noted (McMahon, 1983). Mild and transient abdominal pain was more common with triplicate metrifonate than single dose PZQ ( $75 \%$ versus $30 \%$ ), but the dose of PZQ used (30 mg/ $\mathrm{kg}$ ) was lower than the one currently recommended (40 mg/kg) (WHO, 2002).

\section{Metrifonate: dose comparisons}

One trial with 201 participants compared $3 \times$ $5 \mathrm{mg} / \mathrm{kg}$ metrifonate administered in a single day to $3 \times 7.5 \mathrm{mg} / \mathrm{kg}$ given fortnightly (Aden Abdi and Gustafsson, 1989). There was no significant difference in parasitological failure and egg reduction rate; the geometric mean egg reduction rate was $96 \%$ for the one-day regimen and $97 \%$ for the fortnightly regimen. There was little difference in the percentage of patients with mild adverse events reported for the fortnightly regimen $(7 \%)$ versus the one-day regimen $(9 \%)$.

Three doses of metrifonate $(10 \mathrm{mg} / \mathrm{kg})$ were compared with one and two doses (Rey, Nouhou and Sellin, 1984). There was no significant difference in parasitological failure rates between two and three doses at one month and four months follow-up. Similarly, no significant differences in failure rate and egg reduction rates were detected between two and three doses of $10 \mathrm{mg} / \mathrm{kg}$, given fortnightly in a trial of 81 participants (Rey et al. 1984). By contrast, there were fewer parasitological failures with the threedose regimen over the one dose regimen at the one month follow-up $(\mathrm{RR}=2 \cdot 75,95 \% \mathrm{CI}: 1 \cdot 29-5 \cdot 85$; $n=93$ participants) and the four-month follow-up ( $\mathrm{RR}=1 \cdot 52,95 \% \mathrm{CI}: 1 \cdot 03-2 \cdot 25 ; n=111$ participants).

\section{Different $P Z Q$ doses versus standard regimen $(1 \times 40 \mathrm{mg} / \mathrm{kg})$}

Ten trials compared the standard regimen of $\mathrm{PZQ}$ (single dose of $40 \mathrm{mg} / \mathrm{kg}$ ) to various other doses (McMahon and Kolstrup, 1979; Davis et al. 1981; 
Omer, 1981; Oyediran et al. 1981; Rey et al. 1983; Kardaman et al. 1985; Wilkins and Moore, 1987; Taylor, Murare and Manomano, 1988; King et al. 1989, 2002). In terms of parasitological failure, there was no significant difference between the standard regimen and $2 \times 20 \mathrm{mg} / \mathrm{kg}$ (4 trials), a single dose of $30 \mathrm{mg} / \mathrm{kg}$ (6 trials), and a single dose of $20 \mathrm{mg} / \mathrm{kg}$ (2 trials). Similar results were found at one, three and six months follow-up. Losses to follow-up were generally high, but these did not differ across treatment and control groups within a single trial. There was no significant heterogeneity between the trials, and background endemicity did not seem to play a role. Examining for a differential effect between heavy and moderate or light infections with $30 \mathrm{mg} / \mathrm{kg}$ versus standard $40 \mathrm{mg} / \mathrm{kg}$, a subgroup analysis of one small trial (King et al. 1989) did not show any difference ( $n=116$ participants). Five trials showed no apparent differences in egg reduction rate (geometric mean); all had greater than $95 \%$ reduction in both arms.

\section{Artesunate}

Thus far, only one randomised controlled trial conducted in Gabon in schoolchildren compared the effects of artesunate combined with PZQ to each individual drug given as monotherapy (Borrmann et al. 2001). Whilst the artesunate-PZQ combination resulted in a relatively higher egg reduction rate, it was not possible to identify an effect of artesunate, as no significant difference was observed in cure rates when compared to PZQ alone.

\section{METHODOLOGICAL LIMITATIONS}

Lack of standardization and quality data for the assessment of efficacy and safety of antischistosomal drugs was reported previously for $S$. mansoni (DansoAppiah and de Vlas, 2002). In this Cochrane systematic review (Danso-Appiah et al. 2008), we identified a number of methodological limitations that raise issues with trial quality and the potential for bias, outlined below. Some of the shortcomings have implications for the interpretation of trials in schistosomiasis and other tropical diseases (responses to methodological limitations summarised in Box 1).

(1) Some trials had no proper sample size calculation: this suggests the authors may not have considered whether their study was sufficiently powered to answer the question being posed. (2) Randomisation quality was not high: only four out of 24 trials $(17 \%)$ met quality standards for adequate concealment of allocation and described the methods used (for quality standards see Jüni, Altman and Egger (2001) and Higgins and Green (2008). Trials conducted in the early 1990s and earlier did not conceal allocation except one (Davis et al. 1981). Generation of allocation sequence was adequate in less than
Box 1. Responses to methodological limitations of trials included in a Cochrane review of drugs for treating urinary schistosomiasis (Danso-Appiah et al. 2008)

Design issues

1. There is the need for a unified study methodology in the design, collection and reporting of trials.

2. Trialists should be sensitized to the importance of proper sample size calculation to ensure that trials are sufficiently powered. High losses to follow-up in trials with small sample sizes further compromise the statistical power.

3. There is the need to describe the randomisation procedure clearly.

4. There is a need for standardized, qualitycontrolled diagnostic criteria within and between trials.

5. In high endemicity areas a follow-up time of 4 to 8 weeks is appropriate when investigating cure rates to avoid eggs released from dead worms and minimise the effect of re-infection.

Interpretation and reporting

6. Intensity of infection and egg reduction rate (ERR) should be reported in geometric mean, and intensity of infection should be based on egg count of only the positive cases and reported using the standard classification by the WHO (WHO, 2002).

7. Treatment outcomes need to be clearly defined and standardised across trials. Parasitological outcomes should be reported with; (a) level of edemicity, (b) diagnostic criteria, (c) dose used, (d) age of participants and (e) follow-up time.

half of the trials included in our meta-analysis. For the others, the method used was unclear although all were reported as randomized controlled trials. (3) Losses to follow-up were often high in some trials, and increased proportionally with the duration of follow-up : 17 trials registered losses of $<10 \%$ for short-term evaluations at one to three months, but losses reached up to $50 \%$ in some trials when followup time was longer than three months. (4) Diagnostic criteria were varied, vague and not standardised: among the trials included in our meta-analysis, the criteria for diagnosis varied greatly; some trials used three urine specimens on three consecutive days for microscopic examination, whilst others used a single specimen (Table 1). In some trials sampling criteria varied even between pre- and post-treatment using microscopy (e.g. three urine specimens for the pretreatment diagnosis but only one for post-treatment follow-up assessment) while other trials lacked any criteria for diagnosis. (5) Classification of infection intensities lacked standardization: Table 2 shows considerable variation in the classification of infection 
Table 2. Classification of different S. haematobium infection intensities in clinical trials included in a Cochrane systematic review (Danso-Appiah et al. 2008), n.c. not classified

S. haematobium infection intensity (eggs/10 $\mathrm{ml}$ urine)

\begin{tabular}{llll}
\hline Light & Moderate & Heavy & Reference \\
\hline $60-249$ & $250-499$ & $\geqslant 500$ & Omer (1981) \\
$250-500$ & $501-1000$ & $\geqslant 1000$ & McMahon (1983) \\
$1-5$ & $6-50$ & $>50$ & Rey, Nouhou and Sellin (1984) \\
$1-29$ & $30-99$ & $100-500$ & Stephenson et al. $(1985)$ \\
$1-99$ & $100-399$ & $\geqslant 400$ & King et al. (1988) \\
$<100$ & n.c. & $\geqslant 100$ & Taylor, Murare and Manomano (1988) \\
$1-29$ & $30-99$ & $100-499$ & Stephenson et al. $(1989)$ \\
$1-99$ & $100-399$ & $\geqslant 400$ & King et al. $(1989) ;$ King et al. (2002) \\
$1-49$ & n.c. & $\geqslant 50$ & WHO (2002) \\
\hline \hline
\end{tabular}

intensity across trials. According to current WHO guidelines, infection intensity of $S$. haematobium is either light (1-49 eggs/10 $\mathrm{ml}$ of urine) or heavy $(\geqslant 50$ eggs/10 ml of urine) (WHO, 2002). In the trials included in our meta-analysis, however, light infections were variably classified as 1-5, 1-29, 1-99, 60-249 or even $250-500$ eggs/10 ml of urine. Accordingly, moderate and heavy infections varied from one trial to another. (6) Outcomes were reported in a variety of ways: in our review we defined primary outcomes as (i) parasitological failure and (ii) egg reduction rate. However, these two measures were variably reported as cure rate, failure rate, cumulative failure rate or prevalence for parasitological failure, and as a median, arithmetic mean or geometric mean for egg reduction rate. Even the calculation of geometric mean varied; some investigators considered only the egg-positive individuals, whilst others included the negatives and introduced a correction factor of plus 1 . The latter becomes problematic after treatment when most of the remaining infections are light, as it may overestimate egg count values. (7) Timing of post-treatment assessments varied greatly: the majority of trials evaluated cure and egg reduction rate within one to three months; however, some trials did so at three weeks or earlier, or only six or even 12 months posttreatment. Results from studies on urinary schistosomiasis assessing outcomes earlier than three weeks or beyond three months post-treatment should be considered with caution. The reasons are that the development of $S$. haematobium worms takes approximately two months (Ghandour, 1978); shorter follow-up is confounded by eggs of killed worms still being excreted, longer follow-up by re-infections, particularly in highly endemic settings (N'Goran et al. 2001; Tchuem Tchuenté et al. 2004; Satayathum et al. 2006). Noteworthy, most of the trials in this review were conducted in high endemicity areas and there was no way in differentiating between re-infection and recrudescence.
DISCUSSION

\section{Public health implications}

Despite the above-mentioned methodological limitations, the findings of our Cochrane systematic review have important public health implications. One of the most important findings is that both PZQ and metrifonate are efficacious and safe (DansoAppiah et al. 2008). The failure rate with the recommended standard dose of PZQ $(40 \mathrm{mg} / \mathrm{kg})$ is $0-37 \%$, whilst that of metrifonate $(3 \times 7 \cdot 5-10 \mathrm{mg} / \mathrm{kg}$ given fortnightly) is $19-48 \%$ at one to three months follow-up. However, no trial included in our analysis directly compared the above-mentioned standard doses, therefore precluding any head-to-head assessment of the two treatments from currently available data.

Although the effects of PZQ against placebo are obvious, for some comparisons between regimens with both PZQ and metrifonate there was uncertainty around their effect estimates as shown by the wide $95 \%$ CIs. The small sample size in some of the trials may explain the levels of uncertainty. However, the magnitude of the effect is at times so dramatic that it is unlikely that methodological quality alone will have caused substantive biases to interfere with the marked effects and differences reported.

No difference was demonstrated with a single dose of 20 or $30 \mathrm{mg} / \mathrm{kg}$ of PZQ compared to the standard regimen (single oral dose of $40 \mathrm{mg} / \mathrm{kg}$ ) in terms of all outcomes measured in this review. Given the current emphasis on controlling morbidity in high-burden areas (WHO, 2002), and morbidity, especially among school-aged children, being associated with the number of eggs in an individual, this finding suggests lower doses of PZQ may be effective in morbidity control. However, these results should be considered with caution as detection error can play a role, especially when the studies are few and sample sizes are small. While it is true that parasite load (with egg counts often used as a proxy measure) is an important 
factor in both morbidity for the individual patient and environmental contamination (WHO, 2002), a sub-curative dose may unduly put the drug under selective pressure and favour parasite resistance (Doenhoff, 1998; Doenhoff et al. 2008). Pharmacokinetic data of different doses of PZQ are few and old, and have been obtained in healthy volunteers rather than in schistosome-infected patients (Leopold et al. 1978). An exponential increase was found in the area under the curve (AUC) with the PZQ dose in the range of 5 to $50 \mathrm{mg} / \mathrm{kg}$, with a six-fold increase from 20 to $50 \mathrm{mg} / \mathrm{kg}$ (Leopold et al. 1978). However, these data do not come from infected patients, and hence cannot be extrapolated so easily. This calls for well designed trials incorporating also pharmacokinetic assessment, possibly with sparse sampling and population kinetic assessment. These trials should also control for food intake, as the bioavailability of PZQ depends upon taking it with food and the type of food matters (Mandour et al. 1990; Castro et al. 2000).

The rationale behind the widely spaced dosing intervals of metrifonate treatment derives from its long-lasting effect on red blood cells and plasma cholinesterases (Plestina, Davis and Bailey, 1972). However, the clinical significance of this effect and why side effects disappear during the first 12-24 hours whereas the recovery of the enzymes takes more than 4-6 weeks is not known (Plestina et al. 1972). Safety studies have shown no serious adverse events in patients treated with $5-10 \mathrm{mg} / \mathrm{kg}$ metrifonate daily for 6-12 days (Snellen, 1981), and various reviews of the toxicology and pharmacology of metrifonate during its extensive use for urinary schistosomiasis in the 1970 s concluded that it had very few adverse events (Holmstedt et al. 1978; Feldmeier and Doehring, 1987; Cioli et al. 1995). Also, metrifonate is currently used in Alzheimer's disease in extended regimens, and a systematic review has concluded that overall tolerability is good with only mild to moderate adverse events (LópezArrieta and Schneider, 2006). In the current review, although drug safety was generally poorly reported and assessed in few trials, no trial recorded a serious adverse event, and no significant differences in the number and type of adverse events between metrifonate and PZQ were recorded, except for abdominal pain that was more frequent after metrifonate. Optimizing metrifonate treatment may provide a means of easing drug pressure exerted on schistosomes by the wide deployment of PZQ.

Immature schistosomes are less sensitive to $\mathrm{PZQ}$ than adult worms (Sabah et al. 1986), which has raised concern about controlling schistosomiasis effectively with this drug. Artemisinin derivatives proved to be effective against immature schistosomes in laboratory studies (Utzinger et al. 2003, 2007). However, this review shows that artesunate was not effective against $S$. haematobium infections (though evidence was derived from a single trial (Borrmann et al. 2001)), and combining artesunate and $40 \mathrm{mg} / \mathrm{kg}$ PZQ did not improve efficacy over PZQ alone. In two non-randomised trials involving artesunate alone, results were relatively better (De Clercq et al. 2002; Inyang-Etoh et al. 2004). The latter findings were confirmed in a recent trial; artesunate alone $(4 \mathrm{mg} / \mathrm{kg})$ resulted in a cure rate of $70.5 \%$, whereas an artesunate-PZQ combination obtained a cure rate of $88 \cdot 6 \%$ (Inyang-Etoh et al. 2009). Finally, a recent trial in children under six years of age who were coinfected with Plasmodium falciparum and S. haematobium and who were treated with two different artemisinin-based combinations for malaria therapy showed good effects on S. haematobium. This trial, however, could not be included in the current analysis because there was no control group (Boulanger et al. 2007).

\section{The need for good trial methods}

The validity of randomized controlled trials rests in part on adequate allocation concealment and minimal losses to follow-up, and weaknesses in both these aspects were found in the trials included in the current meta-analysis (Table 1 ). Without adequate allocation concealment properly developed random allocation sequences can be subverted (Schulz and Grimes, 2002). A likely explanation for only four trials $(17 \%)$ included in our final analyses adequately concealing allocation is that this had not been identified as a particularly relevant issue at the time the trials were conducted (20-30 years ago). Even after the publication of the CONSORT statement (Begg et al. 1996) and despite continued educational efforts, the quality of reporting of randomized controlled trials still needs improvement (Altman et al. 2001 ; Moher, Schulz and Altman, 2001).

The effect of losing patients during follow-up on randomisation is crucial as this relates to the internal validity and the power of the trial. In our systematic review we could not do a sensitivity analysis to evaluate the effect of loss to follow-up because data were not sufficient. We encourage trialists to take particular note of this issue and ensure that losses are minimised and power is preserved in future trials. Also, we welcome debate on the most appropriate timing of follow-up in evaluating drug trials of both urinary and intestinal schistosomiasis. The biology of schistosomes suggests that treatment effects with antischistosomal drugs on parasitological parameters should be evaluated during a window of four to eight weeks post-treatment to avoid detecting the tail of eggs released from dead worms on one side, and reinfections on the other side. A first attempt has been made to evaluate this for intestinal schistosomiasis due to $S$. mansoni, and the authors concluded that three weeks after PZQ administration is an appropriate timing for drug efficacy evaluation (Scherrer et al. 2009). 
Safety is generally overlooked and when data are available they are poorly reported. It is important that trialists realise the importance of adequately documenting and reporting on tolerability.

\section{Diagnostic concerns}

The quality of diagnosis can influence the observed cure rates as clearly shown for both $S$. mansoni (de Vlas and Gryseels, 1992; Booth et al. 2003) and S. haematobium (N'Goran et al. 2003). Sensitivity will affect in particular the detection of light infections during follow-up. We found considerable variation in diagnostic criteria not only between, but also within trials, also with regard to infection intensity. This may be explained by the fact that the WHO classification as light (1-49 eggs/10 $\mathrm{ml}$ urine) and heavy infection intensity ( $\geqslant 50$ eggs $/ 10 \mathrm{ml}$ urine) was endorsed only recently (WHO, 2002) and was not in use when the trials summarized here. Because of the different thresholds used for infection intensity, it was not possible to combine and analyse the data according to heavy infections, which is relevant to morbidity control.

\section{Study population issues}

The age of participants enrolled in randomized controlled trials may also influence results. Here, 22 trials out of 24 recruited school-aged children. Hence the overall effect estimates as reported in this review may be lower than studies including all-age subjects, as adults usually show lower infection intensities than school-aged children and, conversely, higher treatment efficacies. This issue has been documented for S. mansoni (Raso et al. 2004), and it is conceivable that the same holds for $S$. haematobium. It should also be noted that restricting treatment to school-aged children leaves untreated adults and pre-school children still excreting eggs to maintain transmission, if indeed transmission is a function of egg output. This brings us to two important sets of considerations. First, data should be reported separately for children and adults before, if necessary, pooling the data to assess overall effects. Second, the purpose of studies depends on the target population. Studies in children receiving antischistosomal treatment are more apt to assess the 'true' efficacy of the drug because drugs such as PZQ have an immune response-dependent component (Doenhoff et al. 1987), which is more active in adults, while whole-population studies are more suited to assess the programmatic effectiveness and effects of control interventions.

IMPLICATIONS FOR POLICY

Both PZQ and metrifonate are effective and safe for treating urinary schistosomiasis. Our systematic review and that of López-Arrieta and Schneider (2006) indicate that metrifonate is well tolerated. Although in schistosomiasis control metrifonate has operational drawbacks, notably multiple administrations, which make it less convenient for largescale morbidity control programmes than a single dose of $\mathrm{PZQ}$, the two drugs have similar efficacy profiles. Furthermore, considering that schistosomes are under intense and growing drug pressure by PZQ and the inherent vulnerability of schistosomiasis control to parasite resistance, we suggest metrifonate should be reconsidered for the treatment of urinary schistosomiasis, to ease the drug pressure on PZQ. This implies continued availability (production and distribution) of the drug. It is also important to have an alternative drug for treating urinary schistosomiasis should PZQ resistance emerge.

Most of the trials contributing to this review were conducted more than a decade ago, and entail a series of methodological limitations. The new schistosomiasis trials must be conducted to contemporary standards of clinical research paying particular attention to quality issues we have raised, and adopt commonly agreed criteria.

Our findings point to new approaches worth being explored in well-designed trials such as: (1) reassessing appropriate dosing schedules for metrifonate, including compliance and feasibility in control programmes; (2) comparing standard metrifonate $(3 \times 7 \cdot 5-10 \mathrm{mg} / \mathrm{kg}$ given fortnightly $)$ and PZQ $(1 \times 40 \mathrm{mg} / \mathrm{kg})$ doses; (3) evaluating artemisinin-based regimens and combination treatments where appropriate (areas where malaria and schistosomiasis are not co-endemic); and (4) obtaining pharmacokinetic/pharmacodynamic correlates for PZQ.

\section{ACKNOWLEDGEMENTS}

We thank Dr. Sake J. de Vlas for providing advice on technical issues. We also thank the Cochrane Infectious Diseases Group (CIDG) in Liverpool for providing administrative support and two anonymous referees for a couple of excellent points. This study received financial support from the Liverpool School of Tropical Medicine, North West Regional Health Authority (UK), Department for International Development (UK), European Commission (Directorate General XII ; Belgium). JU is grateful to the Swiss National Science Foundation for sustained financial support through project no. PPOOB-102883 and PPOOB-119129.

\section{IS C LA IMER}

The opinions expressed in this paper are those of the authors and may not reflect those of their employing organizations. PLO is a staff member of the WHO; the authors alone are responsible for the views expressed in this publication and they do not necessarily represent the decisions, policy or views of the WHO. 
REFERENCES

Aden Abdi, Y. and Gustafsson, L. L. (1989). Field trial of the efficacy of a simplified and standard metrifonate treatments of Schistosoma haematobium. European Fournal of Clinical Pharmacology 37, 371-374.

Altman, D. G., Schulz, K. F., Moher, D., Egger, M., Davidoff, F., Elbourne, D., Gotzsche, P. C. and Lang, T. (2001). The revised CONSORT statement for reporting randomized trials: explanation and elaboration. Annals of Internal Medicine 134, 663-694.

Beasley, N. M. R., Tomkins, A. M., Hall, A., Kihamia, C. M., Lorri, W., Nduma, B., Issae, W., Nokes, C. and Bundy, D. A. P (1999). The impact of population level deworming on the haemoglobin levels of schoolchildren in Tanga, Tanzania. Tropical Medicine and International Health 4, 744-750.

Befidi-Mengue, R. N., Ratard, R. C., Beltran, G., D’Alessandro, A., Rice, J., Befidi-Mengue, R., Kouemeni, L. E. and Cline, B. L. (1992). The impact of Schistosoma haematobium infection and of praziquantel treatment on the growth of primary school children in Bertoua, Cameroon. Yournal of Tropical Medicine and Hygiene 95, 404-409.

Begg, C., Cho, M., Eastwood, S., Horton, R., Moher, D., Olkin, I., Pitkin, R., Rennie, D., Schulz, K. F., Simel, D. and Stroup, D. F. (1996). Improving the quality of reporting of randomized controlled trials. The CONSORT statement. FAMA 276, 637-639.

Bergquist, R., Utzinger, J. and McManus, D. P. (2008). Trick or treat: the role of vaccines in integrated schistosomiasis control. PLoS Neglected Tropical Diseases 2, e244.

Booth, M., Vounatsou, P., N'Goran, E. K., Tanner, M. and Utzinger, J. (2003). The influence of sampling effort and the performance of the Kato-Katz technique in diagnosing Schistosoma mansoni and hookworm co-infections in rural Côte d'Ivoire. Parasitology 127, 525-531.

Borrmann, S., Szlezák, N., Faucher, J.-F., Matsiegui, P.-B., Neubauer, R., Binder, R. K., Lell, B. and Kremsner, P. G. (2001). Artesunate and praziquantel for the treatment of Schistosoma haematobium infections: a double-blind, randomized, placebocontrolled study. Fournal of Infectious Diseases 184, 1363-1366.

Boulanger, D., Dieng, Y., Cisse, B., Remoue, F., Capuano, F., Dieme, J. L., Ndiaye, T., Sokhna, C., Trape, J. F., Greenwood, B. and Simondon, F. (2007). Antischistosomal efficacy of artesunate combination therapies administered as curative treatments for malaria attacks. Transactions of the Royal Society of Tropical Medicine and Hygiene 101, 113-116.

Caffrey, C. R. (2007). Chemotherapy of schistosomiasis: present and future. Current Opinion in Chemical Biology 11, 433-439.

Castro, N., Medina, R., Sotelo, J. and Jung, H. (2000). Bioavailability of praziquantel increases with concomitant administration of food. Antimicrobial Agents and Chemotherapy 44, 2903-2904.

Cioli, D. (2000). Praziquantel: is there real resistance and are there alternatives? Current Opinion in Infectious Diseases 13, 659-663.
Cioli, D., Pica-Mattoccia, L. and Archer, S. (1995). Antischistosomal drugs: past, present ... and future? Pharmacology and Therapeutics 68, 35-85.

Danso-Appiah, A. and de Vlas, S. J. (2002). Interpreting low praziquantel cure rates of Schistosoma mansoni infections in Senegal. Trends in Parasitology 18, 125-129.

Danso-Appiah, A., Utzinger, J., Liu, J. and Olliaro, P. (2008). Drugs for treating urinary schistosomiasis. Cochrane Database Systematic Reviews CD000053.

Davis, A., Biles, J. E., Ulrich, A. M. and Dixon, $\mathbf{H}$. (1981). Tolerance and efficacy of praziquantel in phase II A and II B therapeutic trials in Zambian patients. Arzneimittelforschung 31, 568-574.

De Clercq, D., Vercruysse, J., Kongs, A., Verlé, P., Dompnier, J. P. and Faye, P. C. (2002). Efficacy of artesunate and praziquantel in Schistosoma haematobium infected schoolchildren. Acta Tropica 82, 61-66.

de Vlas, S. J. and Gryseels, B. (1992). Underestimation of Schistosoma mansoni prevalences. Parasitology Today 8, 274-277.

Doehring, E., Ehrich, J. H., Vester, U., Feldmeier, H., Poggensee, U. and Brodehl, J. (1985). Proteinuria, hematuria, and leukocyturia in children with mixed urinary and intestinal schistosomiasis. Kidney International 28, 520-525.

Doenhoff, M. J. (1998). Is schistosomicidal chemotherapy sub-curative? Implications for drug resistance. Parasitology Today 14, 434-435.

Doenhoff, M. J., Cioli, D. and Utzinger, J. (2008). Praziquantel: mechanisms of action, resistance and new derivatives for schistosomiasis. Current Opinion in Infectious Diseases 21, 659-667.

Doenhoff, M. J., Sabah, A. A., Fletcher, C., Webbe, G. and Bain, J. (1987). Evidence for an immune-dependent action of praziquantel on Schistosoma mansoni in mice. Transactions of the Royal Society of Tropical Medicine and Hygiene 81, 947-951.

Feldmeier, H. and Chitsulo, L. (1999). Therapeutic and operational profiles of metrifonate and praziquantel in Schistosoma haematobium infection. Arzneimittelforschung 49, 557-565.

Feldmeier, H. and Doehring, E. (1987). Clinical experience with metrifonate. Review with emphasis on its use in endemic areas. Acta Tropica 44, 357-368.

Feldmeier, H. and Poggensee, G. (1993). Diagnostic techniques in schistosomiasis control. A review. Acta Tropica 52, 205-220.

Fenwick, A., Keiser, J. and Utzinger, J. (2006). Epidemiology, burden and control of schistosomiasis with particular consideration to past and current treatment trends. Drugs of the Future 31, 413-425.

Fenwick, A., Savioli, L., Engels, D., Bergquist, N. R. and Todd, M. H. (2003). Drugs for the control of parasitic diseases: current status and development in schistosomiasis. Trends in Parasitology 19, 509-515.

Finkelstein, J. L., Schleinitz, M. D., Carabin, H. and McGarvey, S. T. (2008). Decision-model estimation of the age-specific disability weight for schistosomiasis japonica: a systematic review of the literature. $P L o S$ Neglected Tropical Diseases 2, e158.

Ghandour, A. M. (1978). The development of Schistosoma haematobium in the hamster. Annals of Tropical Medicine and Parasitology 72, 219-225. 
Higgins, J. P. T. and Green, S. (2008). Cochrane Handbook for Systematic Reviews of Interventions. Version 5.0.1. The Cochrane Collaboration, 2008 (available from www.cochrane-handbook.org).

Holmstedt, B., Nordgren, I., Sandoz, M. and Sundwall, A. (1978). Metrifonate. Summary of toxicological and pharmacological information available. Archives of Toxicology 41, 3-29.

Inyang-Etoh, P. C., Ejezie, G. C., Useh, M. F. and Inyang-Etoh, E. C. (2004). Efficacy of artesunate in the treatment of urinary schistosomiasis, in an endemic community in Nigeria. Annals of Tropical Medicine and Parasitology 98, 491-499.

Inyang-Etoh, P. C., Ejezie, G. C., Useh, M. F. and Inyang-Etoh, E. C. (2009). Efficacy of a combination of praziquantel and artesunate in the treatment of urinary schistosomiasis in Nigeria. Transactions of the Royal Society of Tropical Medicine and Hygiene 103, 38-44.

Jewsbury, J. M. and Cooke, M. J. (1976). Prophylaxis of schistosomiasis - field trial of metrifonate for the prevention of human infection. Annals of Tropical Medicine and Parasitology 70, 361-363.

Jia, T. W., Zhou, X. N., Wang, X. H., Utzinger, J., Steinmann, P. and Wu, X. H. (2007) Assessment of the age-specific disability weight of chronic schistosomiasis japonica. Bulletin of the World Health Organization 85, 458-465.

Jinabhai, C. C., Taylor, M., Coutsoudis, A., Coovadia, H. M., Tomkins, A. M. and Sullivan, K. R. (2001) Epidemiology of helminth infections: implications for parasite control programmes, a South African perspective. Public Health Nutrition 4, 1211-1219.

Jüni, P., Altman, D. G. and Egger, M. (2001). Systematic reviews in health care: assessing the quality of controlled clinical trials. BMY 323, 42-46.

Kardaman, M. W., Fenwick, A., el Igail, A. B., el Tayeb, M., Daffalla, A. A. and Dixon, H. G. (1985). Treatment with praziquantel of schoolchildren with concurrent Schistosoma mansoni and S. haematobium infections in Gezira, Sudan. Fournal of Tropical Medicine and Hygiene 88, 105-109.

King, C. H. and Bertino, A. M. (2008). Asymmetries of poverty: why global burden of disease valuations underestimate the burden of neglected tropical diseases. PLoS Neglected Tropical Diseases 2, e209.

King, C. H., Dickman, K. and Tisch, D. J. (2005). Reassessment of the cost of chronic helmintic infection: a meta-analysis of disability-related outcomes in endemic schistosomiasis. Lancet 365, 1561-1569.

King, C. H., Lombardi, G., Lombardi, C., Greenblatt, R., Hodder, S., Kinyanjui, H., Ouma, J., Odiambo, O., Bryan, P. J., Muruka, J., Magak, P., Weinert, D., Mackay, W., Ransohoff, D., Houser, H., Koech, D., Siongok, T. K. A. and Mahmoud, A. A. F. (1988). Chemotherapy-based control of schistosomiasis haematobia. I. Metrifonate versus praziquantel in control of intensity and prevalence of infection. American Fournal of Tropical Medicine and Hygiene 39, 295-305.

King, C. H., Muchiri, E. M., Mungai, P., Ouma, J. H., Kadzo, H., Magak, P. and Koech, D. K. (2002).

Randomized comparison of low-dose versus standard-dose praziquantel therapy in treatment of urinary tract morbidity due to Schistosoma haematobium infection. American Fournal of Tropical Medicine and Hygiene 66, 725-730.

King, C. H., Wiper, D. W. 3rd, De Stigter, K. V., Peters, P. A. S., Koech, D., Ouma, J. H., Arap Siongok, T. K. and Mahmoud, A. A. F. (1989). Dose-finding study for praziquantel therapy of Schistosoma haematobium in Coast Province, Kenya. American Fournal of Tropical Medicine and Hygiene 40, 507-513.

Leopold, G., Ungethum, W., Groll, E., Diekmann, H. W., Nowak, H. and Wegner, D. H. (1978). Clinical pharmacology in normal volunteers of praziquantel, a new drug against schistosomes and cestodes. An example of a complex study covering both tolerance and pharmacokinetics. European Fournal of Clinical Pharmacology 14, 281-291.

López-Arrieta, J. M. and Schneider, L. (2006). Metrifonate for Alzheimer's disease. Cochrane Database Systematic Reviews CD003155.

Mandour, M. E., el Turabi, H., Homeida, M. M., el Sadig, T., Ali, H. M., Bennett, J. L., Leahey, W. J. and Harron, D. W. (1990). Pharmacokinetics of praziquantel in healthy volunteers and patients with schistosomiasis. Transactions of the Royal Society of Tropical Medicine and Hygiene 84, 389-393.

McMahon, J. E. (1983). A comparative trial of praziquantel, metrifonate and niridazole against Schistosoma haematobium. Annals of Tropical Medicine and Parasitology 77, 139-142.

McMahon, J. E. and Kolstrup, N. (1979). Praziquantel: a new schistosomicide against Schistosoma haematobium. British Medical Fournal 2 (6202), 1396-1398.

Moher, D., Schulz, K. F. and Altman, D. G. (2001). The CONSORT statement: revised recommendations for improving the quality of reports of parallel-group randomized trials. Annals of Internal Medicine 134, 657-662.

N'Goran, E. K., Utzinger, J., Gnaka, H. N., Yapi, A., N'Guessan, N. A., Kigbafori, S. D., Lengeler, C., Chollet, J., Xiao, S. H. and Tanner, M. (2003). Randomized, double-blind, placebo-controlled trial of oral artemether for the prevention of patent Schistosoma haematobium infections. American fournal of Tropical Medicine and Hygiene 68, 24-32.

N'Goran, E. K., Utzinger, J., N'Guessan, A. N., Müller, I., Zamble, K., Lohourignon, K. L., Traoré, M., Sosthene, B. A., Lengeler, C. and Tanner, M. (2001). Reinfection with Schistosoma haematobium following school-based chemotherapy with praziquantel in four highly endemic villages in Côte d'Ivoire. Tropical Medicine and International Health $\mathbf{6}$, $817-825$

Olds, G. R., King, C., Hewlett, J., Olveda, R., Wu, G., Ouma, J., Peters, P., McGarvey, S., Odhiambo, O., Koech, D., Liu, C. Y., Aligui, G., Gachihi, G., Kombe, Y., Parraga, I., Ramirez, B., Whalen, C., Horton, R. J. and Reeve, P. (1999). Double-blind placebo-controlled study of concurrent administration of albendazole and praziquantel in schoolchildren with schistosomiasis and geohelminths. Fournal of Infectious Diseases 179, 996-1003. 
Omer, A. H. (1981). Praziquantel in the treatment of mixed $S$. haematobium and $S$. mansoni infections. Arzneimittelforschung 31, 605-608.

Oyediran, A. B., Kofie, B. A., Bammeke, A. O. and Bamgboye, E. A. (1981). Clinical experience with praziquantel in the treatment of Nigerian patients infected with S. haematobium. Arzneimittelforschung 31, 581-584.

Plestina, R., Davis, A. and Bailey, D. R. (1972). Effect of metrifonate on blood cholinesterases in children during the treatment of schistosomiasis. Bulletin of the World Health Organization 46, 747-759.

Pugh, R. N. H. and Teesdale, C. H. (1983). Single dose oral treatment in urinary schistosomiasis: a double blind trial. British Medical Fournal 286, 429-432.

Raso, G., N'Goran, E. K., Toty, A., Luginbühl, A., Adjoua, C. A., Tian-Bi, N. T., Bogoch, I. I., Vounatsou, P., Tanner, M. and Utzinger, J. (2004). Efficacy and side effects of praziquantel against Schistosoma mansoni in a community of western Côte d'Ivoire. Transactions of the Royal Society of Tropical Medicine and Hygiene 98, 18-27.

Rey, J. L., Nouhou, H. and Sellin, B. (1984). Comparaison de trois posologies de metrifonate en chimiotherapie de masse contre Schistosoma haematobium. Médecine Tropicale 44, 57-60.

Rey, J. L., Sellin, B., Gazere, O., Ott, D., Reges, M. and Garrouty, P. (1983). Comparaison au Niger de l'efficacite sur Schistosoma haematobium du praziquantel (30 mg/kg et $40 \mathrm{mg} / \mathrm{kg}$ ) en une prise et de l'oltipraz $(35 \mathrm{mg} / \mathrm{kg})$ en deux prises. Medecine et Maladies Infectieuses 13, 328-331.

Sabah, A. A., Fletcher, C., Webbe, G. and Doenhoff, M. J. (1986). Schistosoma mansoni: chemotherapy of infections of different ages. Experimental Parasitology 61, 294-303.

Satayathum, S. A., Muchiri, E. M., Ouma, J. H., Whalen, C. C. and King, C. H. (2006). Factors affecting infection or reinfection with Schistosoma haematobium in coastal Kenya: survival analysis during a nine-year, school-based treatment program. American Fournal of Tropical Medicine and Hygiene 75, 83-92.

Scherrer, A. U., Sjöberg, M. K., Allangba, A., Traoré, M., Lohourignon, L. K., Tschannen, A. B., N'Goran, E. K. and Utzinger, J. (2009).

Sequential analysis of helminth egg output in human stool samples following albendazole and praziquantel administration. Acta Tropica 109, 226-231.

Schulz, K. F. and Grimes, D. A. (2002). Allocation concealment in randomised trials : defending against deciphering. Lancet 359, 614-618.
Snellen, W. M. (1981). Therapeutic properties of metrifonate. Acta Pharmacologica et Toxicologica 49 (Suppl. 5), 114-117.

Steinmann, P., Keiser, J., Bos, R., Tanner, M. and Utzinger, J. (2006). Schistosomiasis and water resources development: systematic review, metaanalysis, and estimates of people at risk. Lancet Infectious Diseases 6, 411-425.

Stephenson, L. S., Kinoti, S. N., Latham, M. C., Kurz, K. M. and Kyobe, J. (1989). Single dose metrifonate or praziquantel treatment in Kenyan children. I. Effects on Schistosoma haematobium, hookworm, hemoglobin levels, splenomegaly, and hepatomegaly. American Fournal of Tropical Medicine and Hygiene 41, 436-444.

Stephenson, L. S., Latham, M. C., Kurz, K. M., Kinoti, S. N., Oduori, M. L. and Crompton, D. W. T. (1985). Relationships of Schistosoma haematobium, hookworm and malarial infections and metrifonate treatment to growth of Kenyan school children. American Fournal of Tropical Medicine and Hygiene 34, 1109-1118.

Taylor, P., Murare, H. M. and Manomano, K. (1988). Efficacy of low doses of praziquantel for Schistosoma mansoni and S. haematobium. Fournal of Tropical Medicine and Hygiene 91, 13-17.

Tchuem Tchuenté, L. A., Shaw, D. J., Polla, L., Cioli, D. and Vercruysse, J. (2004). Efficacy of praziquantel against Schistosoma haematobium infection in children. American Fournal of Tropical Medicine and Hygiene 71, 778-782.

The Cochrane Collaboration (2003). Review Manager (RevMan) 4.2 for Windows. The Nordic Cochrane Centre, Copenhagen.

Utzinger, J. and Keiser, J. (2004). Schistosomiasis and soil-transmitted helminthiasis : common drugs for treatment and control. Expert Opinion on Pharmacotherapy 5, 263-285.

Utzinger, J., Keiser, J., Xiao, S. H., Tanner, M. and Singer, B. H. (2003). Combination chemotherapy of schistosomiasis in laboratory studies and clinical trials. Antimicrobial Agents and Chemotherapy 47, 1487-1495.

Utzinger, J., Xiao, S. H., Tanner, M. and Keiser, J. (2007). Artemisinins for schistosomiasis and beyond. Current Opinion in Investigational Drugs 8, 105-116.

WHO (2002). Prevention and control of schistosomiasis and soil-transmitted helminthiasis : report of a WHO expert committee. WHO Technical Repoort Series No. 912, 1-57.

Wilkins, H. A. and Moore, P. J. (1987). Comparative trials of regimes for the treatment of urinary schistosomiasis in The Gambia. Fournal of Tropical Medicine and Hygiene 90, 83-92. 